Trauma Berufskrankh 2015 • [Suppl 2]:

17:338-344

DOI 10.1007/s10039-015-0023-7

Online publiziert: 4. Juni 2015

๑) Springer-Verlag Berlin Heidelberg 2015

Isabella Mehling · Michael Sauerbier

Abteilung für Plastische, Hand- und Rekonstruktive Chirurgie - Hand-Trauma-Center,

Berufsgenossenschaftliche Unfallklinik Frankfurt am Main, Frankfurt am Main, Deutschland

\title{
Knöcherne Verletzungen der Handwurzel
}

Durch eine forcierte Extension, aber auch durch eine Ulnarduktion des Handgelenkes kommt es zu einer dorsalen Subluxation des Mediokarpalgelenkes und somit zu einer vermehrten Belastung auf die palmare Kortikalis des Skaphoids.

In $60 \%$ der Fälle betrifft die Fraktur das mittlere Skaphoiddrittel, zu jeweils $15 \%$ das proximale und distale Skaphoiddrittel und zu $10 \%$ das Tuberculum ossis scaphoidei [29].

Nach dem Konzept von Lichtman fungiert die Handwurzel als ein unter Spannung stehender Ring [8]. Kommt es zu einer ligamentären oder knöchernen Verletzung kann eine Unterbrechung des Ringes resultieren. Die Aufhebung des Ringgefüges durch eine instabile Skaphoidfraktur führt langfristig zu einer karpalen Instabilität bis hin zum karpalen Kollaps [4, 7]. Die karpale Achsenfehlstellung erzeugt eine typische Instabilität und Fehlstellung, die sog. DISI („dorsiflexed intercalated segment instability")-Gefügestörung, bei der das Lunatum zusammen mit dem proximalen Skaphoidpol nach dorsal in die Extensionsstellung kippt und das distale Skaphoidfragment gegenläufig in die palmare Flexionsstellung. Im weiteren Verlauf kommt es zu einer periskaphoidalen Arthrosis deformans bis zum fortgeschrittenen karpalen Kollaps mit Ausbildung eines sog. „scaphoid nonunion advanced collapse (SNAC) wrist" $[13,15]$.

Die Gefäßversorgung des Skaphoids erfolgt für das Tuberculum ossis scaphoidei und den distalen 20- bis 30 \%igen Skaphoidanteil aus den palmaren Ästen der A. radialis sowie des daraus hervorgehenden R. palmaris superficialis. Die proximalen $70-80 \%$ des Skaphoids erhalten ihre Gefäßversorgung über die dorsale Knochenkante. Da der proximale Pol keine eigene, suffiziente Blutversorgung auf- weist, treten nach unbehandelten Skaphoidfrakturen im proximalen Drittel 3- bis 6-mal häufiger Pseudarthrosen und ggf. auch avaskuläre Nekrosen auf [23, 28, 32].

Bei klinischem Verdacht einer Skaphoidfraktur muss eine radiologische Abklärung erfolgen, bis die Skaphoidfraktur entweder festgestellt oder aber ausgeschlossen wurde. Standardmäßig erfolgt zunächst eine Handgelenkaufnahme im posterior-anterioren und seitlichen Strahlengang. Aufgrund der gekrümmten Form des Skaphoids sowie der gekippten Lage von $45^{\circ} \mathrm{zu}$ den 3 orthogonalen Raumebenen kommt es nur zu einer unvollständigen Darstellung. Zur vollständig einsehbaren Skaphoidbetrachtung ist die Zusatzaufnahme in Faustschluss und Ulnarduktion nach „Stecher" hilfreich [17, 33].

In der traditionellen Röntgendiagnostik erfolgt zusätzlich die Kahnbein-Quartettserie, die die Aufnahme nach Stecher (Faustschluss und Ulnarduktion), nach Schreck (Schreibfederstellung), nach Bridgeman (Extensionsstellung) und in Hyperpronation umfasst [28]. Dies ist aktuell jedoch nicht mehr erforderlich, da bei Frakturverdacht immer eine hochauflösende CT-Untersuchung des Kahnbeins erfolgen sollte.

\section{CT-Untersuchung}

Standardmäßig sollte jedoch zur weiteren Abklärung hinsichtlich der Detektion und der möglichen therapeutischen Konsequenzen eine hochauflösende Computertomographie (CT) durchgeführt werden. Hierdurch kann eine exakte Klassifikationseinteilung erfolgen, was wichtig für die Behandlungsentscheidung ist.

Die CT-Untersuchung sollte parallel zur Längsachse des Skaphoids mit einer 


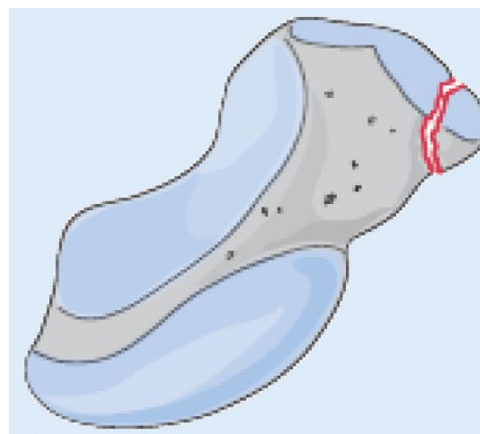

A1

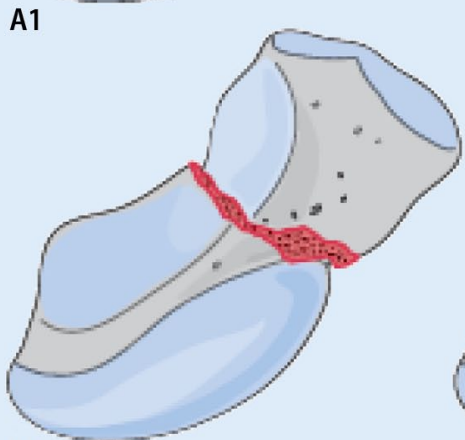

B2

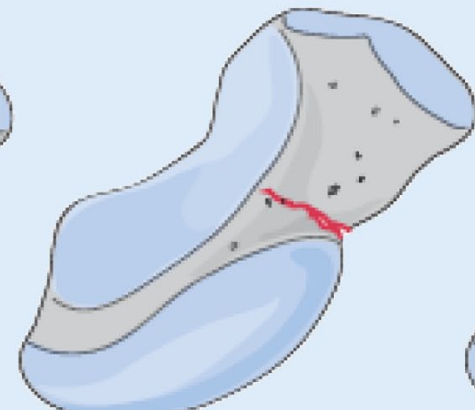

A2
B3

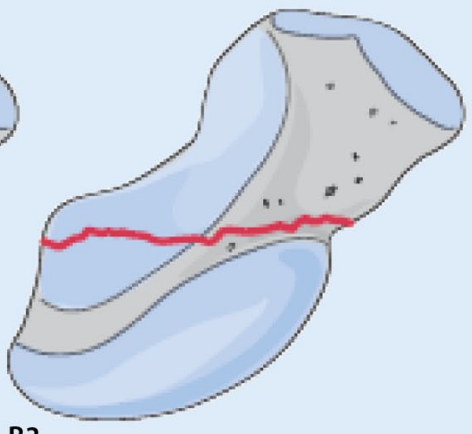

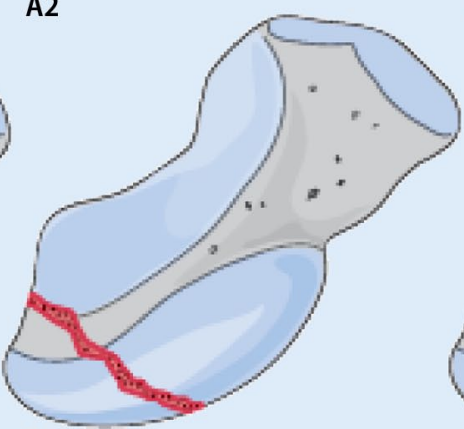

B3

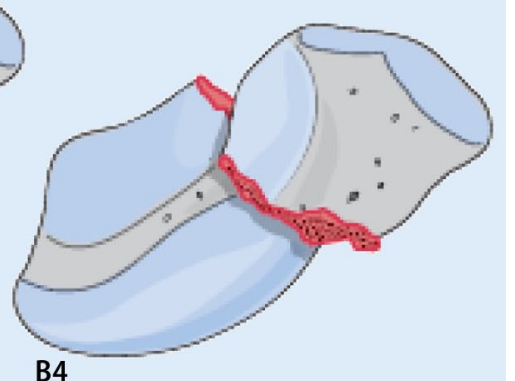

B4
Abb. $1<$ Klassifikation der frischen Skaphoidfrakturen, mod. nach Krimmer/Herbert [17]. (Mit freundl. Genehmigung des Thieme Verlages, [20])
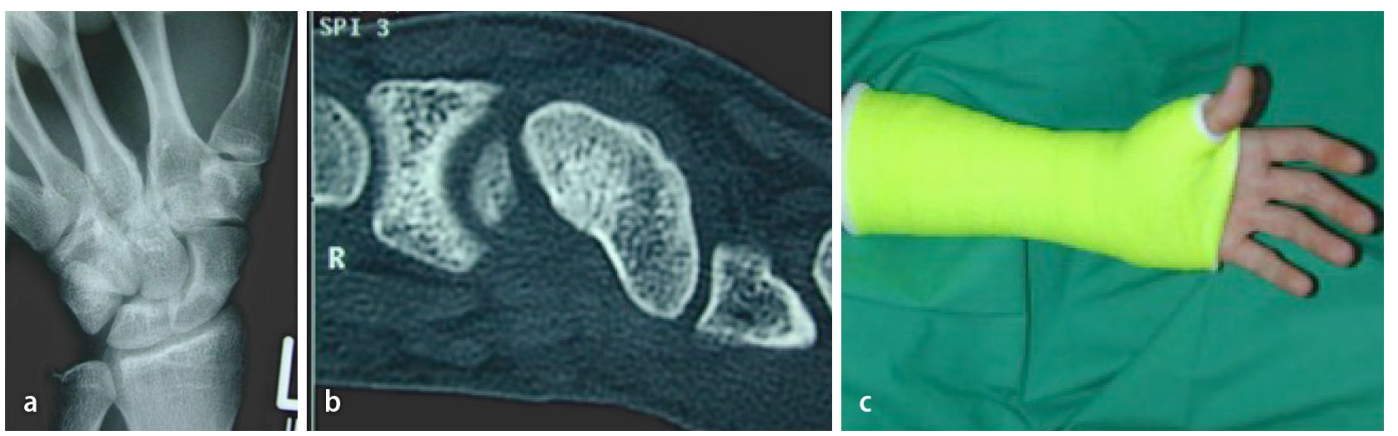

Abb. $2<16$-jähriger Patient mit Sturz auf das linke Handgelenk. a In der konventionellen Röntgendiagnostik in posterior-anterior ist die Skaphoidfraktur kaum sichtbar. b Die CT-Aufnahme in sagittaler Schnittführung zeigt die stabile A2-Fraktur. c Konservative Therapie mittels Skaphoidcast mit Daumengrundgelenkeinschluss

Schichtdicke von 0,5-1 mm erfolgen. Damit die Scanebene parallel zur Längsausdehnung des Skaphoids verläuft, muss die Hand in einem Winkel von $45^{\circ}$ zur Körperachse gelagert werden. Mit dieser Untersuchung können kleinste Dislokation oder auch Trümmerzonen sehr gut aufgedeckt werden [14, 30]. Mit State-ofthe-Art-Scannern, die annähernd isotrope Voxel erzeugen (Schichtdicke 0,4-0,6 mm, Bildmatrix größer als 1024 x 1024) kann ein Volumendatensatz der gesamten Handwurzel erstellt werden, sodass die schräg-sagittalen und schräg-koronalen Dünnschichten durch das Skaphoid rekonstruiert werden können [30].

\section{MRT-Untersuchung}

Die Magnetresonanztomographie (MRT) kann ebenfalls eine Skaphoid- fraktur nachweisen. Dabei sollten die Schichtbilder in gleicher Projektion erfolgen wie bei der CT-Untersuchung. Im MRT kommen in der Frühphase nach dem Unfallereignis traumatisierte Bereiche als Knochenmarködem signalintensiv zur Darstellung. Jedoch kann dabei nicht immer zwischen einer vollständigen Fraktur und einem Knochenmarködem unterschieden werden. Daher ist zur Darstellung und genauen Beurteilung der Frakturzone die CT-Untersuchung vorzuziehen. Besteht jedoch der Verdacht einer okkulten Fraktur, vor allem bei Kindern, so sollte zunächst eine MRT-Untersuchung erfolgen. Zur Differenzierung einer Nekrose am proximalen Pol oder auch bei Morbus Preiser sollte ebenfalls eine MRT mit Gadolinium durchgeführt werden.

\section{Einteilung der Skaphoidfrakturen}

Die Einteilung der Skaphoidfrakturen erfolgt anhand der nativ-radiologischen Bildgebung nach der Einteilung von Herbert [11]. Da routinemäßig zur exakten Frakturbeurteilung eine CT-Untersuchung parallel zur Längsachse des Skaphoids erfolgen sollte, wurde die ursprünglich beschriebene Klassifikation nach Herbert mit zusätzlicher CT-Beurteilung nach Krimmer/Herbert modifiziert ([17; • Abb. 1):

- Typ A: stabile Frakturen

- A1: Fraktur des Tuberculum ossis scaphoidei

- A2: nicht dislozierte oder inkomplette Fraktur im mittleren/distalen Drittel

- Typ B: instabile Frakturen 
- B1: Schrägfraktur

- B2: dislozierte Fraktur

- B3: Fraktur im proximalen Drittel

- B4: transskaphoidale perilunäre Luxationsfraktur

Die Klassifikation unterscheidet dabei zwischen stabilen (Typ A) und instabilen (Typ B) Frakturen. Prinzipiell sollten alle instabilen Frakturen (Typ B1-B4) aufgrund der hohen Pseudarthrosengefahr operativ versorgt werden.

Es besteht vor allem bei proximalen Skaphoidfrakturen (Typ B3) ein erhöhtes Risiko für Pseudarthrose und für das Auftreten von avaskulären Nekrosen, was daran liegt, dass dem proximalen Skaphoidabschnitt eine eigenständige Blutversorgung fehlt.

Bei der Typ-B4-Fraktur handelt es sich um eine transskaphoidale perilunäre $\mathrm{Lu}$ xationsfraktur und somit um eine komplexe Verletzung mit zur Fraktur zusätzlichen Läsionen der karpalen Ligamente.

Stabile Skaphoidfrakturen (Typ A1-A2 in der CT-Klassifikation nach Krimmer/ Herbert) können in einem Skaphoidgips ausbehandelt werden. Beim Skaphoidgips handelt es sich um einen Unterarmgips bzw. Unterarmcast mit Einschluss des Daumengrundgelenkes (• Abb. 2). Die früher häufig durchgeführte Immobilisation des Ellenbogengelenkes durch einen Oberarmgips hat keinen nachgewiesenen Vorteil und ist verzichtbar $[6,16,24,35]$. Die Ruhigstellung sollte für 6 bis 8 Wochen erfolgen mit anschließender Röntgenkontrolle in den 3 Standardebenen. Wir empfehlen zudem die Durchführung einer erneuten Röntgen (Stecher)- und CT-Untersuchung nach 6-wöchiger Ruhigstellung zum Nachweis der knöchernen Durchbauung [21]. Zeigt sich spätestens nach 12-wöchiger Ruhigstellung noch keine suffiziente Frakturdurchbauung, so sollte eine operative Therapie geplant werden [10].

Stabile A2-Skaphoidfrakturen können alternativ auch operativ versorgt werden, wenn der Patient dies wünscht, um schneller wieder seinen Alltagsaktivitäten nachkommen zu können, sowie keine längere Ruhigstellung wünscht. Nach minimalinvasiver Skaphoidosteosynthese empfehlen wir eine Ruhigstellungsdauer von ca. 1 Woche. Der Vergleich zwischen konservativer Therapie und minimalinvasivem Operationsverfahren zeigte, dass das operative Vorgehen eine schnellere knöcherne Durchbauung und damit auch eine frühere Arbeits- und Sportfähigkeit ermöglicht $[3,6,9,19]$. Die Ergebnisse der Studien zeigen, dass die operative Therapie die besten Ergebnisse hinsichtlich Funktion und frühzeitiger Rückkehr zum Arbeitsplatz ermöglicht. Allerdings scheinen die Komplikationen etwas höher zu sein als nach konservativer Therapie [26].

Alle als instabil klassifizierten frischen Skaphoidfrakturen sollten operativ versorgt werden. Ziel der operativen Therapie ist eine stabile Frakturversorgung, die eine frühe Mobilisation und somit auch eine schnelle Rückkehr in das Arbeitsleben ermöglicht sowie die Ausbildung einer Pseudarthrose vermeidet.

Durch die Einführung der HerbertSchraube 1984 konnte die operative Versorgung von Skaphoidfrakturen entscheidend verbessert werden [11]. Sowohl die ursprüngliche Herbert-Schraube als auch die heute gebräuchlichen kanülierten Titanschrauben verfügen über ein Spitzenund ein Kopfgewinde (Doppelgewindeschraube), deren beider Durchmesser größer ist als der des gewindefreien Schraubenschaftes. Um eine axiale Kraft auf die Fraktur zu erreichen, ist die Gewindesteigung im Kopf geringer als an der Spitze, sodass es zu einer Kompression kommt, sobald beide Gewinde im Knochen liegen und die Schraube weiter angezogen wird. Die Schraube sollte vollständig intraossär und sicher subchondral platziert werden. Dadurch ist bei regelrechter Schraubenlage eine Materialentfernung nach knöcherner Konsolidierung meist nicht notwen$\operatorname{dig}[20]$.

Die operative Technik zur Skaphoidstabilisierung richtet sich nach der Frakturlokalisation, dem Ausmaß und der Richtung der Dislokation, eventuellen begleitenden Verletzungen und den Erfahrungen und Vorlieben des Operateurs [22, 25]. Die Versorgung von Frakturen im proximalen Drittel erfolgt üblicherweise von dorsal. Frakturen im mittleren und distalen Drittel werden meist von palmar in offener oder minimalinvasiver Technik operiert, sie können jedoch auch
Trauma Berufskrankh $2015 \cdot[$ Suppl 2]: 17:338-344

DOI 10.1007/s10039-015-0023-7

(c) Springer-Verlag Berlin Heidelberg 2015

\section{Mehling $\cdot$ M. Sauerbier KnöcherneVerletzungen der Handwurzel}

\section{Zusammenfassung}

Knöcherne Verletzungen der Handwurzel betreffen zu $80 \%$ das Skaphoid und zu $15 \%$ das Triquetrum. Frakturen der übrigen Handwurzelknochen sind deutlich seltener. Eine frühzeitige CT-Diagnostik hat nicht nur für die Skaphoidfraktur, sondern auch bezüglich der anderen Handwurzelknochen einen hohen Stellenwert in der Erkennung einer Fraktur sowie der nachfolgenden Therapiemaßnahme. Stabile karpale Frakturen können erfolgreich konservativ therapiert werden. Dislozierte karpale Frakturen sollten osteosynthetisch versorgt werden. Ziele sind die anatomische Reposition sowie eine stabile Osteosynthese. Hierdurch kommt es in den meisten Fällen zu einer Ausheilung unter Wiederherstellung der Funktion des Handgelenkes.

\section{Schlüsselwörter}

Handwurzelfrakturen · Skaphoid .

Triquetrum - Computertomographie . Osteosynthese

\section{Injuries of the carpal bones}

\section{Abstract}

In fractures involving the carpal bones, $80 \%$ are fractures of the scaphoid bone and $15 \%$ involve the triquetrum bone. Fractures of the remaining carpal bones are exceedingly rare. A prompt computed tomography (CT) scan is required for detecting not only fractures of the scaphoid bone but also other carpal bone fractures and for determining an adequate therapy. Undisplaced fractures can be successfully treated conservatively. Displaced or unstable carpal bone fractures should be operatively treated with open reduction and internal fixation, whereby bone union and restoration of range of motion and hand function can be achieved..

\section{Keywords}

Carpal bone fractures - Scaphoid bone . Triquetrum bone - Computed tomography . Osteosynthesis

von dorsal operativ angegangen werden (• Abb. 3).

Der Algorithmus für die Behandlung einer Skaphoidfraktur ist in $\bullet$ Abb. 4 dargestellt. Skaphoidfrakturen können auch als Teil einer karpalen Luxationsfraktur 

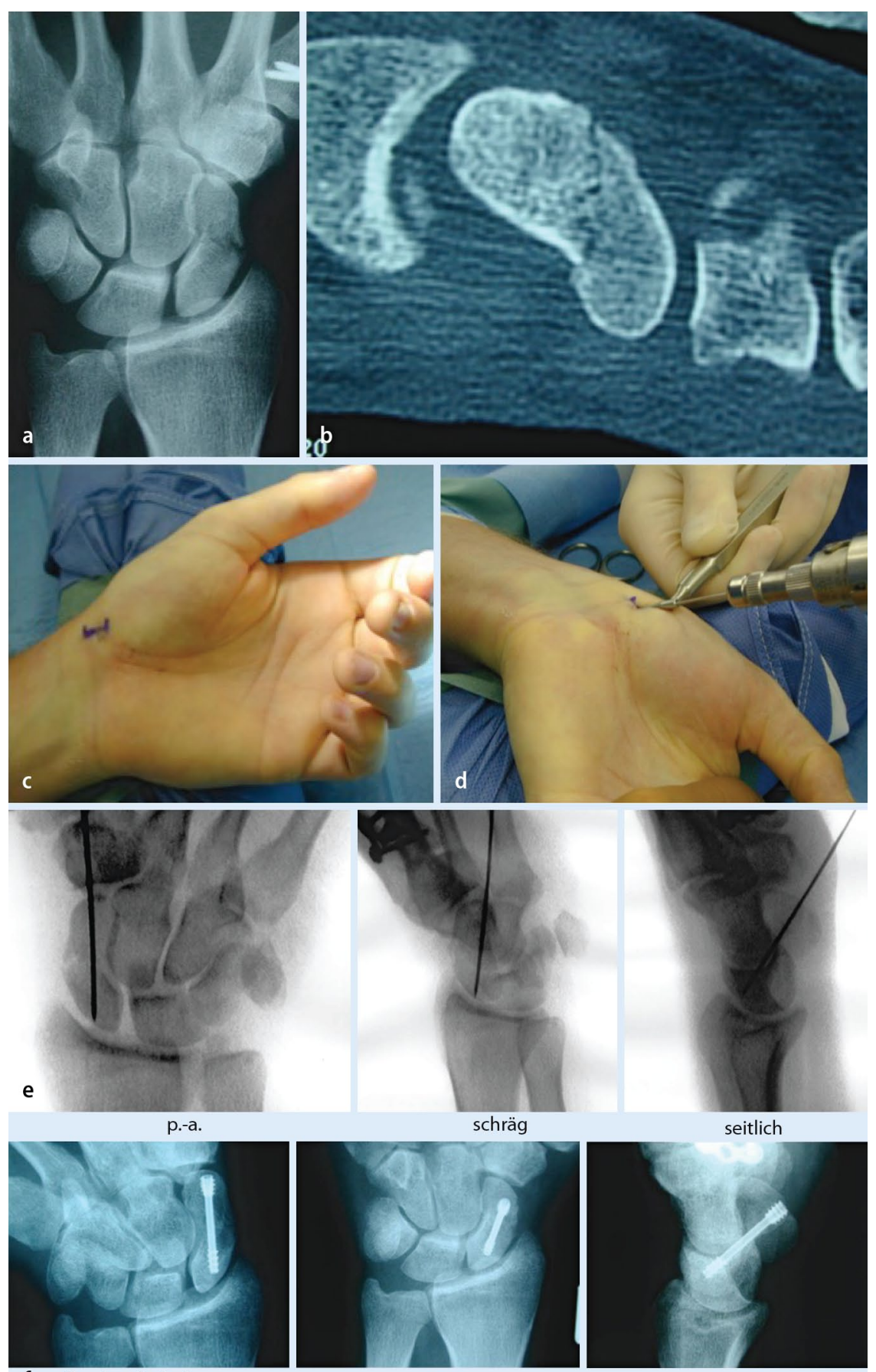

f Stecher-Aufnahme
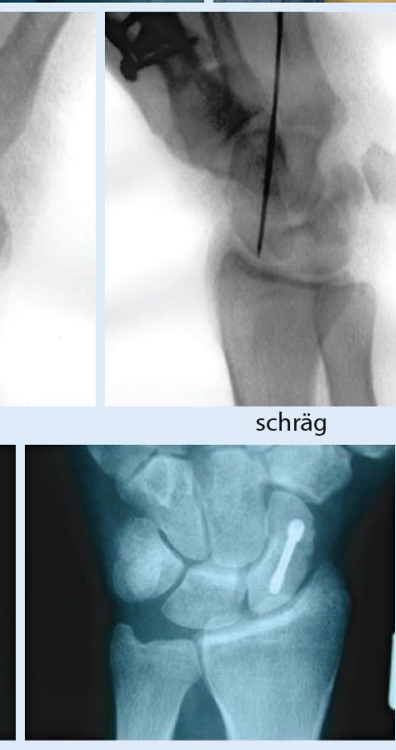

p.-a.

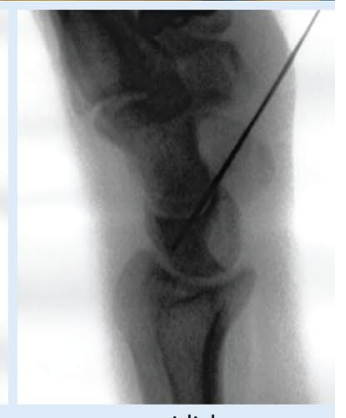

seitlich

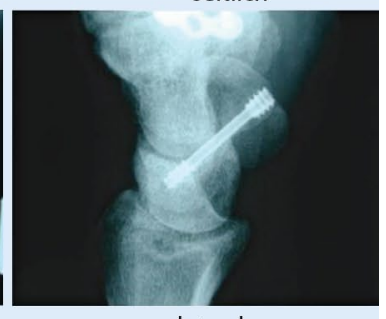

lateral

Abb. 3 \& 32-jähriger Patient mit Sturz auf das linke Handgelenk. a, b Im Nativröntgen sowie in der CT-Aufnahme sichtbare Skaphoidfraktur Typ B1. c, d Inzision palmar über dem STT (Skaphoid-Trapezium-Trapezoideum)-Gelenk, Anbringen der Bohrdrahthülse und Vorbohren eines 1,0-mm-Kirschner-Drahtes. e Intraoperative Röntgenkontrolle zur Drahtlage in posterior-anteriorem (p.-a.), schrägem und seitlichem Strahlengang, dann bei regelrechter Lage Einbringen einer kanülierten HerbertSchraube. f Postoperative Röntgenkontrolle der regelrechten Herbert-Schrauben-Lage in der StecherAufnahme, im p.-a.- und seitlichen Strahlengang

vorkommen. Dabei handelt es sich um seltenere, aber besonders schwere Verletzungen, die meist bei einem Hochrasanztrauma oder einem Sturz aus größerer Höhe auftreten. Die typische Luxations-

einer Ruptur des lunotriquetralen Bandes und letztendlich zu einer Dislokation des Lunatum $[18,32]$. Man unterscheidet zwischen Verrenkungslinien des kleinen Bogens (,lesser arc injury") und denen entlang des großen Bogens (,greater arc injury"). Bei Letzteren kann die Luxationslinie praktisch durch alle Handwurzelknochen verlaufen, wobei Skaphoid, Kapitatum und Triquetrum am häufigsten betroffen sind. Die perilunäre Luxation mit Fraktur des Skaphoids wird als "transskaphoidale perilunäre Luxationsfraktur" (de Quervain) bezeichnet. Diese ist mit $61 \%$ die häufigste Form der perilunären Luxationen $[29,32]$.

\section{Weitere karpale Frakturen}

Frakturen der übrigen Handwurzelknochen sind sehr selten und machen ca. 1,1\% aller Frakturen aus. Der Unfallmechanismus ist wie bei der Skaphoidfraktur ein Sturz auf die ausgestreckte Hand [27, 34]. Eine große Bedeutung für die Detektion der Handwurzelfrakturen hat heutzutage die CT-Diagnostik.

\section{Triquetrumfraktur}

Triquetrumfrakturen machen ca. $15 \%$ aller Karpusfrakturen aus und stellen damit die zweithäufigsten knöchernen Verletzungen des Karpus dar. Die Triquetrumfrakturen werden in Frakturen der dorsalen Kortikalis (Chipfrakturen, Korpusfrakturen) und palmare Avulsionsfrakturen eingeteilt.

Die Behandlung der frischen Triquetrumchip- oder Avulsionsfrakturen erfolgt meist konservativ mit einer Ruhigstellung für 3 bis 4 Wochen. Eine dislozierte Korpusfraktur sollte operativ versorgt werden, z. B. mittels offener Reposition und Osteosynthese durch kanülierte Minischrauben.

\section{Trapeziumfraktur}

Bei ca. 1-5\% aller Karpusfrakturen handelt es sich um Trapeziumfrakturen. Dabei kommt es meist durch eine axiale Kompression auf den Daumen zu einer vertikalen, intraartikulären Fraktur.

Nicht dislozierte Frakturen werden konservativ behandelt mittels Ruhigstel- 


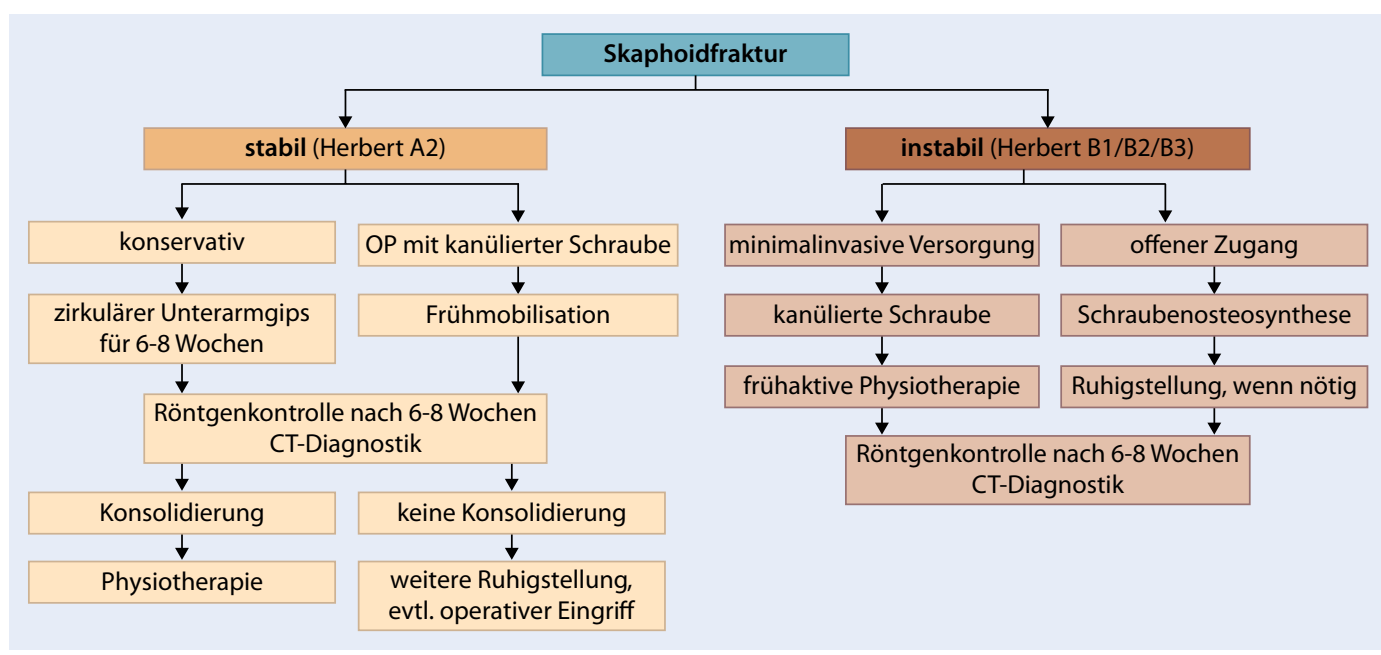

Abb. $4<$ Algorithmus für die Behandlung der frischen Skaphoidfraktur [20]. (Mit freund. Genehmigung des Thieme Verlages) lung für maximal 4 Wochen. Dislozierte Frakturen sollten operativ therapiert werden mittels geschlossener oder offener Reposition und Stabilisierung mittels Draht- oder Schraubenosteosynthese, da sonst die Gefahr einer Daumensattel- oder Skaphoid-Trapezium-Trapezoideum-Gelenkarthrose besteht (• Abb. 5).

\section{Kapitatumfraktur}

Kapitatumfrakturen treten isoliert nur in $1-2 \%$ aller Handwurzelfrakturen auf. Meist handelt es sich dabei um quer verlaufende Korpusfrakturen. Häufiger treten Kapitatumfrakturen in Verbindung mit einer perilunären Verletzung auf. Hier ist vor allem das „scaphocapitate syndrome" nach Fenton zu nennen, bei dem es sich um eine transskaphoidale transkapitale perilunäre Luxation handelt, die häufig mit Rotation des proximalen Kapitatumfragments bei Hochrasanztrauma vorliegt [1].

Nicht dislozierte Frakturen werden konservativ behandelt und heilen in der Regel nach 6 Wochen aus. Dislozierte Frakturen sollten operativ versorgt werden, da vor allem nach übersehener, dislozierter Fraktur die Gefahr einer Pseudarthrosenausbildung besteht. Zudem kann bei diesem Frakturtyp eine Nekrose am Kapitatumkopf entstehen, die zur mediokarpalen Arthrose und nachfolgend zum karpalen Kollaps führen kann [27, 34].

\section{Lunatumfraktur}

Lunatumfrakturen sind mit 0,5-1\% aller Karpusfrakturen selten. Meist besteht eine quer verlaufende Lunatumkorpusfraktur. Die Lunatumfrakturen werden in eine Fraktur des palmaren Pols, des distalen Pols sowie in eine transverse, osteochondrale und transartikuläre Korpusfraktur unterteilt. Eine operative Therapie ist lediglich bei den Karpus-destabilisierenden Randfrakturen und dislozierten Korpusfrakturen indiziert.

Differenzialdiagnostisch ist immer an eine Lunatumnekrose zu denken und diese auszuschließen. Hierbei ist bei einer traumatisch bedingten Lunatumfraktur anamnestisch ein adäquates Trauma zu fordern. In der bildgebenden Diagnostik (Röntgen-, CT- und auch MRT-Untersuchung) zeigen sich Zeichen einer frischen Frakturierung, ggf. auch eine Mitbeteiligung der benachbarten Carpalia, dagegen keine Zeichen einer Osteonekrose.

\section{Hamatumfraktur}

Hamatumfrakturen machen ca. $2 \%$ aller Karpusfrakturen aus. Es werden dabei Korpusfrakturen von den Hamulusfrakturen unterschieden. Die Klassifikation nach Hirano und Inoue unterscheidet in Typ-1- und Typ-2-Frakturen [12]:

\section{Typ 1: Hamulusfrakturen}

Unverschobene Hamulusfrakturen können konservativ therapiert werden. Eine konservative Therapie hat dann die besten Erfolgsaussichten, wenn sie innerhalb von maximal 3 Wochen nach Trauma begonnen wird. Die Ruhigstellung sollte für 6 Wochen erfolgen und den Hamulus durch $15^{\circ}$ Flexion und $5^{\circ}$ Radialduktion des Handgelenkes entlasten [5].

Eine operative Therapie ist bei frischen Frakturen fakultativ und individuell mit dem Patienten zu besprechen. Bei veralteten Frakturen sowie bei schmerzhaften Pseudarthrosen sollte eine operative Therapie erfolgen [5]. Die operative Therapie kann zum einen durch eine Schraubenosteosynthese des Hamulus erfolgen, zum anderen durch die Exstirpation des Hamulus. In beiden Fällen besteht das Risiko einer Verletzung des motorischen Astes (R. profundus) des N. ulnaris. Dabei sollte diese Operation möglichst vom handchirurgisch Erfahrenen unter Lupenbrillenvergrößerung durchgeführt werden.

\section{Typ 2: Frakturen des Korpus}

2a Koronale Frakturen bei CMC (karpometakarpale)-Luxationsfraktur des 4. und 5. Strahls. Da die Reposition problemlos gelingt besteht die Therapie in einer geschlossenen Reposition und einer perkutanen Kirschner-Draht-Transfixation.

2b Transversale Frakturen. Eine isolierte Querfraktur des Hamatumkorpus ist sehr selten. Die Therapie sollte operativ mittels Reposition und Osteosynthese mit einer Doppelgewindeschraube erfolgen (• Abb. 6). 

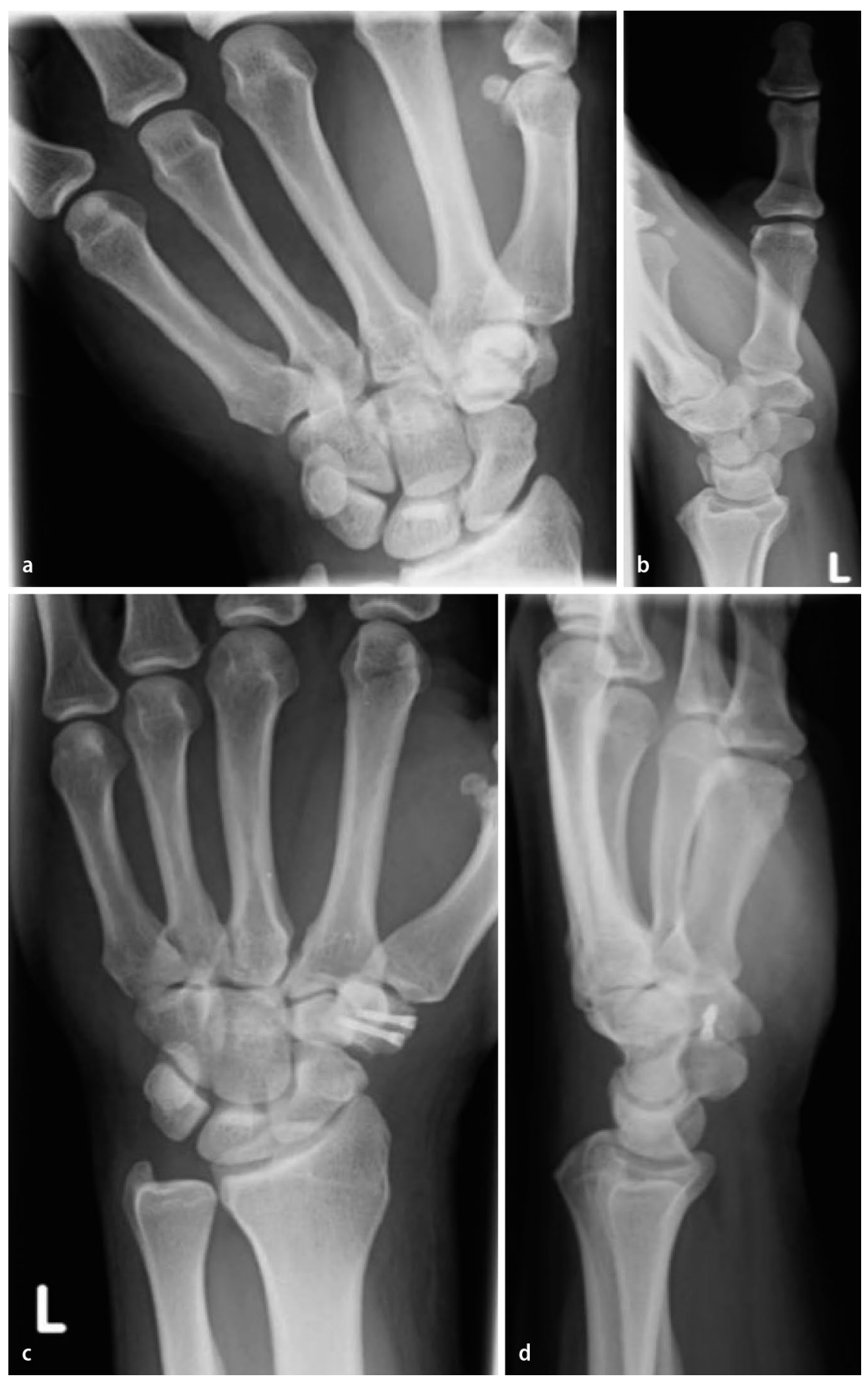

Abb. $5 \Delta$ a, b Dislozierte Trapeziumfraktur. c, d Operative Versorgung mittels 2 Minischrauben

\section{Pisiformefraktur}

Frakturen des Os pisiforme machen ca. $2 \%$ aller Handwurzelfrakturen aus. Meist handelt es sich um ein direktes Trauma mit Sturz auf die ulnare Handwurzel. Bei den Verletzungen des Os pisiforme wird zwischen Frakturen und Luxationen unterschieden.
Bei persistierenden Beschwerden ist ggf. eine Exzision des Os pisiforme möglich ohne relevante Nachteile für die Handfunktion $[2,27,34]$.

\section{Trapezoideumfraktur}

Trapezoideumfrakturen kommen bei knöchernen Handwurzelverletzungen aufgrund der Lage und durch den Schutz kräftiger Bandverbindungen am seltensten vor und machen unter $1 \%$ der Karpusfrakturen aus. Sie sind meist Folge eines indirekten Traumas im Rahmen einer karpometakarpalen Luxationsfraktur. Nicht oder wenig dislozierte Frakturen werden konservativ behandelt. Bei starker Dislokation, vor allem bei karpometakarpaler Luxationsfraktur sollte eine geschlossene oder offene Reposition und Osteosynthese durch Drähte und/oder Minischraube durchgeführt werden [27, 34].

Bei persistierender Fehlstellung und Instabilitäten ist ggf. eine karpometakarpale Arthrodese indiziert.

\section{Fazit für die Praxis}

- Frakturen der Handwurzel betreffen zu $80 \%$ das Skaphoid. Die adäquate Diagnostik und Therapie der Skaphoidfraktur spielen daher im klinischen Alltag die größte Rolle bei Handwurzelverletzungen. Die frühzeitige CTDiagnostik hat einen hohen Stellenwert in der Erkennung der Handwurzelfrakturen.

- Stabile karpale Frakturen können erfolgreich konservativ therapiert werden. Dislozierte karpale Frakturen sollten osteosynthetisch versorgt werden, wobei minimalinvasive Osteosynthesen immer häufiger mit kanülierten Schrauben durchgeführt werden. Arthroskopisch assistierte Operationen können bei der Reposition förderlich sein. Dadurch ist auch eine Behandlung bzw. der Ausschluss von ligamentären Zusatzverletzungen möglich. Durch eine anatomische Reposition und stabile Osteosynthese kommt es in den meisten Fällen zu einer Ausheilung unter Wiederherstellung der Funktion des Handgelenkes. 

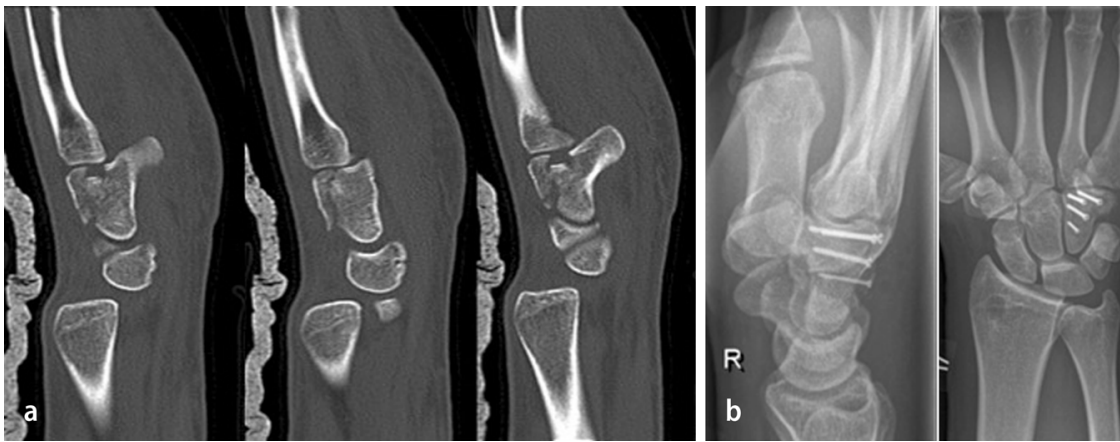

Abb. $6 \Delta$ a Impressionsfraktur Os hamatum. b Offene Reposition der Fraktur, autologe Spongiosaplastik von der distalen Speiche und Osteosynthese mit 4 Schrauben $(3 \times 1,7 \mathrm{~mm}, 1 \times 1,2 \mathrm{~mm})$

\section{- Komplexe knöcherne Handwurzelver- letzungen fallen unter das Schwerst- verletzungsartenverfahren der Hand und sollten daher nur in handchirur- gischen Spezialabteilungen behan- delt werden. \\ - Ziel der Behandlung ist die Wieder- herstellung der anatomischen Form mit Erhalt einer schmerzfreien Hand- gelenkfunktion sowie die Wieder- erlangung der vollständigen Arbeits- fähigkeit. Spätfolgen nach knöcher- nen Handwurzelfrakturen, die nicht primär erkannt bzw. nicht adäquat versorgt wurden, münden nicht sel- ten in eine Minderung der Erwerbs- fähigkeit.}

\section{Korrespondenzadresse}

\section{Priv. Doz. Dr. I. Mehling}

Abteilung für Plastische, Hand- und Rekonstruktive Chirurgie - Hand-Trauma-Center Berufsgenossenschaftliche Unfallklinik Frankfurt am Main

Friedberger Landstraße 430

60389 Frankfurt am Main

isabellamaria.mehling@bgu-frankfurt.de

\section{Einhaltung ethischer Richtlinien}

Interessenkonflikt. I. Mehling und M. Sauerbier geben an, dass kein Interessenkonflikt besteht.

Dieser Beitrag beinhaltet keine Studien an Menschen oder Tieren.

The supplement containing this article is not sponsored by industrie.

\section{Literatur}

1. Arbter D, Piatek S, Wichlas F et al (2009) Die scaphokapitale Fraktur der Han (Fenton). Handchir Mikrochir Plast Chir 41:171-174
2. Arner M, Hagberg L (1984) Wrist flexion strength after excision of the pisiform bone. Scand J Plast Reconstr Surg 18:241-245

3. Arora R, Gschwentner M, Krappinger D et al (2007) Fixation of nondisplaced scaphoid fractures: making treatment cost effective. Prospective controlled trial. Arch Orthop Trauma Surg 127:39-46

4. Baumeister HH, Greinemann H (1989) Zur konservativen Behandlung des Kahnbeinbruches der Handwurzel. Unfallchirurg 92:175-179

5. Bickert B (2014) Hamatumfrakturen - Diagnostik und Therapie. Handchirurgie Scan 3:229-237

6. Bond CD, Shin AY, Mcbride MT et al (2001) Percutaneous screw fixation or cast immobilization for nondisplaced scaphoid fractures. J Bone Joint Surg Am 83-A:483-488

7. Brauer RB, Dierking M, Werber KD (1997) Die Anwendung der Herbert-Schraube mit der FrehandMethode zur Osteosynthese der frischen Skaphoidfraktur. Unfallchirurg 100:776-781

8. Brown DE, Lichtman DM (1987) Midcarpal instability. Hand Clin 3:135-140

9. Davis EN, Chung KC, Kotsis SV et al (2006) A cost/ utility analysis of open reduction and internal fixation versus cast immobilization for acute nondisplaced mid-waist scaphoid fractures. Plast Reconstr Surg 117:1223-1235; (discussion 12361228)

10. Dias JJ, Taylor M, Thompson J et al (1988) Radiographic signs of union of scaphoid fractures. An analysis of inter-observer agreement and reproducibility. Bone Joint Surg Br 70:299-301

11. Herbert TJ, Fisher WE (1984) Management of the fractured scaphoid using a new bone screw. Bone Joint Surg Br 66:114-123

12. Hirano K, Inoue G (2005) Classification and treatment of hamate fractures. Hand Surg 10:151-157

13. Krakauer JD, Bishop AT, Cooney WP (1994) Surgical treatment of scapholunate advanced collapse. J Hand Surg 19:751-759

14. Krimmer H (2010) Kahnbeinfraktur- Diagnostik und Therapie - aktueller Stand. Obere Extremität 5(2):98-105

15. Krimmer $\mathrm{H}$ (2002) Management of acute fractures and nonunions of the proximal pole of the scaphoid. J Hand Surg Br 27:245-248

16. Krimmer $H$, Sauerbier M, Vispo-Seara JL et al (1992) Fortgeschrittener karpaler Kollaps (SlacWrist) bei Skaphoidpseudarthrose - Therapiekonzept: Mediokarpale Teilarthrodese. Handchir Mikrochir Plast Chir 24:191-198

17. Krimmer H, Schmitt R, Herbert $T$ (2000) Kahnbeinfraktur - Diagnsotik, Klassifikation und Therapie. Unfallchirurg 103:812-819
18. Mayfield JK, Johnson RP, Kilcoyne RK (1980) Carpal dislocations: pathomechanics and progressive perilunar instability. J Hand Surg 5:226-241

19. Mcqueen MM, Gelbke MK, Wakefield A et al (2008) Percutaneous screw fixation versus conservative treatment for fractures of the waist of the scaphoid: a prospective randomised study. J Bone Joint Surg Br 90:66-71

20. Mehling IM, Sauerbier M (2013) Skaphoidfrakturen und Skaphoidpseudarthrosen. Z Orthop Unfall 151:639-660

21. Müller M, Chen ZB, Al Morshidy A et al (2006) Ergebnisse frischer osteosynthetisch verschraubter Skaphoidfrakturen nach postoperativer CT-Kontrolluntersuchung. Unfallchirurg 109:185-192

22. Müller M, Germann G, Sauerbier M (2008) Minimal invasive screw fixation and early mobilization of acute scaphoid fractures in the middle third: operative technique and early functional outcome. Tech Hand Up Extrem Surg 12:107-113

23. Reigstad O, Grimsgaard C, Thorkildsen R et al (2012) Scaphoid non-unions, where do they come from? The epidemiology and initial presentation of 270 scaphoid non-unions. Hand Surg 17:331-335

24. Saeden B, Tornkvist H, Ponzer S et al (2001) Fracture of the carpal scaphoid. A prospective, randomised 12-year follow-up comparing operative and conservative treatment. J Bone Joint Surg Br 83:230-234

25. Sauerbier M, Müller M (2007) Skaphoidfrakturen: Diagnostik, Zugangswege, Komplikationen. Zentralbl Chir 132:W42-W53; (quiz W54-45)

26. Schädel-Höpfner M, Marent-Huber M, Sauerbier M et al (2010) Operative vs. konservative Therapie nicht dislozierter Skaphoidfrakturen. Ergebnisse einer kontrollierten multizentrischen Kohortenstudie. Unfallchirurg 113(804):806-813

27. Schädel-Höpfner $M$, Prommersberger KJ, Eisenschenk A et al (2010) Behandlung von Handwurzelfrakturen. Empfehlungen der Sektion Handchirurgie der Deutschen Gesellschaft für Unfallchirurgie. Unfallchirurg 113:741-754 (quiz 755)

28. Schmidt HU, Lanz U (2003) Chirurgische Anatomie der Hand. Thieme, Stuttgart

29. Schmitt R, Lanz U (2003) Bildgebende Diagnostik der Hand. Thieme, Stuttgart Bitte einfügen, neue Literaturstelle 30 :

30. Schmitt R, Lanz U (2015) Bildgebende Diagnostik der Hand. Georg Thieme Verlag KG, Stuttgart New York

31. Schmitt RFS (2004) Computertomographie in Bildgebende Diagnostik der Hand. In: Schmitt RLU (Hrsg) Thieme, Stuttgart, S 63-68

32. Slutsky DJ, Slade JF 3rd (2011) The scaphoid. Thieme, Stuttgart

33. Stecher W (1937) Roentgenography of the carpal navicular bone. Am J Roentgenol 704-705

34. Suh N, Ek ET, Wolfe SW (2014) Carpal fractures. J Hand Surg 39:785-791 (quiz 791)

35. Vinnars B, Pietreanu M, Bodestedt A et al (2008) Nonoperative compared with operative treatment of acute scaphoid fractures. A randomized clinical trial. J Bone Joint Surg Am 90:1176-1185 